\title{
Practice Spotlight: Pharmacists in an Antimicrobial Stewardship Program
}

\author{
Linda Dresser, PharmD \\ Pharmacotherapy Specialist - \\ Antimicrobial Stewardship \\ University Health Network \\ Assistant Professor \\ Leslie Dan Faculty of Pharmacy \\ University of Toronto \\ Toronto, Ontario \\ Sandra Nelson, BSP, ACPR, PharmD \\ Clinical Practice Leader - \\ Antimicrobial Stewardship \\ Mount Sinai Hospital \\ Toronto, Ontario
}

T hree years ago, the Infectious Diseases Society of America (IDSA) and the Society for Healthcare Epidemiology of America (SHEA) published guidelines for developing an institutional antimicrobial stewardship program. ${ }^{1}$ One impetus for these guidelines was emerging evidence that many hospitals do not actively work to ensure that antimicrobials are used appropriately, despite well-described problems with antimicrobial resistance and nosocomial infections with organisms that have been linked to antimicrobial overuse, such as Clostridium difficile. Another reason for developing and disseminating antimicrobial stewardship guidelines was the paucity of new antimicrobials in the drug development pipeline, as documented by the IDSA. ${ }^{2}$

Antimicrobial stewardship is the practice of using antimicrobials appropriately to optimize patient outcomes, minimize costs, and avoid the adverse effects and collateral damage associated with these drugs. The IDSA-SHEA guidelines recommend that the core members of an antimicrobial stewardship program include (but not be limited to) an infectious diseases physician and a pharmacist with infectious diseases training and that the program be appropriately supported by the institution's senior administration. ${ }^{1}$ The guidelines also recommend prospective audit (with interventions and feedback) and restriction and preauthorization of the formulary as key stewardship activities. ${ }^{1}$
The antimicrobial stewardship programs at Mount Sinai Hospital and the University Health Network in Toronto, Ontario, were launched in February and September 2009, respectively. These programs are essentially a joint initiative of the 2 institutions, with the same infectious diseases physician as director of both, but separate administrative funding. A business case was presented to senior administrators at each institution, outlining the needs and scope of the proposed programs, to obtain appropriate institutional support. The 2 programs are well funded, both financially and in terms of human resources. In addition to the physician director, the programs together employ a second infectious diseases physician (at the University Health Network), a total of 3 pharmacists (1 at Mount Sinai Hospital and 2 at the University Health Network, including Linda Dresser and Sandra Nelson), 2 project managers, and 2 data analysts ( 1 of each at each institution). Additionally, members of the programs work closely with the other key personnel identified in the IDSA-SHEA guidelines: microbiologists, infection control practitioners, and information technologists.

The 3 pillars of the antimicrobial stewardship programs at Mount Sinai Hospital and the University Health Network are clinical practice, education, and research. Pharmacists perform essential aspects of the activities of all 3 pillars on a daily basis.

Clinical practice currently involves specific initiatives of prospective audit and feedback in 3 patient care areas: intensive care units (ICUs), general surgery, and leukemia services. The pharmacists and/or physician of the antimicrobial stewardship programs meet with the medical teams on these services 3 to 5 times weekly, depending on the service, to review the clinical situation for patients who are receiving antimicrobial therapy and to provide suggestions on appropriate use of these drugs when needed. In addition to the inpatient program at the University Health Network, the pharmacists and physician of the antimicrobial stewardship programs have developed and initiated an outpatient parenteral antimicrobial therapy clinic, which provides care to patients who have been discharged from hospital but who are still receiving IV antimicrobial therapy. As part of this outpatient service, one of the pharmacists assesses the appropriateness and safety of selected antimicrobial therapy before discharge. Other clinical activities under development include the formulation, in collaboration with the key stake- 
holders and front-line staff (e.g., ICU physicians, pharmacists, and nurses), of best practice guidance for certain clinical syndromes.

Education, which is fundamental to the success of an antimicrobial stewardship program, occurs in many forums and in a variety of formats. For example, meetings involving a pharmacist from the antimicrobial stewardship program and members of the targeted teams to review appropriateness of antimicrobials represent opportunities to provide education on a variety of antimicrobial and infectious diseases topics. In addition, the antimicrobial stewardship team provides education at an institutional level via rounds, newsletters, and reports, and to multiple audiences such as peers, students, residents, and hospital administrators.

A successful antimicrobial stewardship program will be able to utilize the data it creates to demonstrate outcomes not only for the institution but also for the broader health care audience. Therefore, each initiative of the stewardship programs at Mount Sinai Hospital and the University Health Network is linked to a research question. At present, the data generated relate predominantly to continuous quality and safety improvements. The program at Mount Sinai has been in operation for longer than a year and has already achieved significant decreases in antimicrobial costs, usage, and-most importantly-resistance. For example, in the hospital's ICU, yearly costs for antimicrobials have decreased by about 30\%, and usage of these drugs has declined by about $15 \%$. The susceptibility of isolates of Pseudomonas spp. to antimicrobials has increased by $5 \%-17 \%$, depending on the agent.

Future endeavours will include expansion of the prospective audit and feedback service by the program's pharmacists to other areas of the hospital, such as internal medicine, cardiovascular care, and orthopedic surgery. Research will continue on quality improvement initiatives, and additional research will be undertaken focusing on identified areas for which minimal evidence currently exists. Educational goals include the development of a stewardship training program for physicians and pharmacists. The members of the antimicrobial stewardship team will continue to follow their mantra in all that they do: "Getting patients the right antimicrobial, when they need it."

\section{References}

1. Dellit TH, Owens RC, McGowan JE Jr, Gerding DN, Weinstein RA, Burke JP, et al. Infectious Diseases Society of America and the Society of Healthcare Epidemiology of America guidelines for developing an institutional program to enhance antimicrobial stewardship. Clin Infect Dis 2007;44(2):159-177.

2. Bad bugs, no drugs: as antibiotic discovery stagnates . . a public health crisis brews. Alexandria (VA): Infectious Diseases Society of America; 2004 Jul [cited 2010 Jun 22]. Available from: https:/www.idsociety.org/ 10x20.htm

The Practice Spotlight series highlights the accomplishments of Canadian pharmacists with unique practices in hospitals and related health care settings. If you have a unique or innovative practice, or you know someone else who should be profiled, please submit your contact information to Mary Ensom, Editor of CJHP (cjhpedit@cshp.ca), and one of our Associate Editors will be in touch with you. 